\title{
The Evolution of Genomic Imprinting
}

\author{
Atsushi Mochizuki, Yasuhiko Takeda and Yoh Iwasa \\ Department of Biology, Faculty of Science, Kyushu University, Fukuoka 812-81, Japan
}

Manuscript received November 24, 1995

Accepted for publication July 30, 1996

\begin{abstract}
In some mammalian genes, the paternally and maternally derived alleles are expressed differently: this phenomenon is called genomic imprinting. Here we study the evolution of imprinting using multivariate quantitative genetic models to examine the feasibility of the genetic conflict hypothesis. This hypothesis explains the observed imprinting patterns as an evolutionary outcome of the conflict between the paternal and maternal alleles. We consider the expression of a zygotic gene, which codes for an embryonic growth factor affecting the amount of maternal resources obtained through the placenta. We assume that the gene produces the growth factor in two different amounts depending on its parental origin. We show that genomic imprinting evolves easily if females have some probability of multiple partners. This is in conflict with the observation that not all genes controlling placental development are imprinted and that imprinting in some genes is not conserved between mice and humans. We show however that deleterious mutations in the coding region of the gene create selection against imprinting.
\end{abstract}

$\mathrm{S}^{\mathrm{E}}$ EVERAL mammalian embryonic genes are known to be subject to genomic imprinting. Only either the paternally or maternally derived allele is actively expressed while the other stays silent (PETERSON and SAPIENZA 1993). Some of these imprinted genes code for an embryonic growth factor or its inhibitor and therefore affect placental development, while others are related to suckling and swallowing behaviors, appetite, and attracting maternal attention. It has been suggested that imprinted genes are likely to be involved in controlling the amount of resources supplied by the mother (HAIG and Graham 1991; MOORE and HaIG $1991)$.

Genomic imprinting was first noted from the growth retardation and death of embryos that possessed the maternal duplication and paternal deficiency (or paternal duplication and maternal deficiency) of the whole genome, or of a single chromosome (uniparental disomy). FERGUSON-SMITH et al. (1991) incorporated cells with paternal duplication of the distal chromosome 7 into chimeras and found a considerable growth enhancement in the embryos. In contrast, in embryos with maternal duplication of the distal chromosome 7 , both the maternal alleles of the $\operatorname{Ig} f 2$ (insulin-like growth factor type-2) gene were repressed. The $\lg f 2$ gene in this region was suspected to be imprinted. Using gene targeting, DeCHIARA et al. (1991) demonstrated that the paternally inherited disrupted $\operatorname{Ig} f 2$ gene causes growth deficiency but the maternally inherited disrupted gene does not. Nuclease protection and in situ hybridization analyses of the transcripts from the wild-

Corresponding author: Atsushi Mochizuki, Department of Biology, Faculty of Science, Kyushu University, Fukuoka 812-81, Japan.

E-mail: amochscb@mbox.nc.kyushu-u.ac.jp type and mutated alleles indicated that only the paternal allele is expressed in embryos, while the maternal allele is silent. An exception is found in the choroid plexus and leptomeninges, where both the maternal and paternal $\operatorname{Ig} f 2$ genes are expressed. Similarly, only the paternally derived alleles are expressed for several genes on human chromosome 15, which are in turn related to behavioral abnormalities, such as Prader-Willi syndrome and Angelman syndrome (OzCELIK et al. 1992; SUTCLIFF et al. 1994; WEvRICK et al. 1994).

In contrast, there are genes in which only the maternally derived copy is actively expressed. For example, $\lg f 2 r$ (insulin-like growth factor type-2 receptor) is expressed only from the maternal chromosome in mice (BARLOW et al. 1991). This has been suspected to be an inhibitor of $I g f 2$ (HAIG and GRAHAM 1991). BARTOLOMEI et al. (1991) showed that in the mouse the H19 gene is imprinted, with the active copy derived from the mother. H19 RNA has tumor-suppressor activity (HAO et al. 1993), but it is, however, not translated. Some genes controlling the cell cycle are also imprinted in the mouse: an inhibitor of the cyclin / CDK complex, $\mathrm{p} 57^{\mathrm{KIP2}}$, is transcribed only from the maternal allele (HATADA and MUKAI 1995), while an activator gene, CDC25, is transcribed only from the paternal allele in some tissues (Plass et al. 1996).

Control of differential genetic expression is known to be based on the methylation pattern of the genes (SASAKI et al. 1993). ChAillet et al. (1991) studied methylation of transgenes and found that both maternally and paternally inherited methylation patterns are erased in primordial germ cells and that distinctive patterns emerge during germ cell maturation. The methylation pattern found in sperm undergoes further modification during embryogenesis. SASAKI et al. (1991) 
examined whether the parental-origin-dependent differential methylation observed in transgenes reflects the genomic imprinting of endogenous genes, and confirmed that the methylation patterns are established before early prophase I during spermatogenesis. UEDA et al. (1992) showed that parental-specific adult patterns of transgene methylation are established during gametogenesis. Li et al. (1993) examined expression of three imprinted genes $(\operatorname{Ig} f 2, \operatorname{Ig} f 2 r, H 19)$ in mutant mice that are deficient in DNA methyltransferase activity. Both maternal and paternal genes of H19 are expressed, but both genes are repressed for $\operatorname{Ig} f 2$ and $\operatorname{Ig} f 2 r$.

Recent studies have revealed that the genetically imprinted genes, Mash-2, Ins-2, Igf2, and H19, are clustered into a chromosomal domain, implying that imprinting may be regulated in a regional matter (EDEN and CEDAR 1995). In a similar vein, a group of genomically imprinted human genes ( $S N R P N, P A R-5, P A R-$ $1, I P W)$ is located within a single region of several hundred kb (OzCELIK et al. 1992; WevRICK et al. 1994) and is suspected to be controlled by an imprinting-controlling element (Reis et al. 1994; SUTCLIFF et al. 1994; BUITING et al. 1995).

Genomic imprinting of a particular gene may differ between species, between individuals of the same species, and between tissues of the same individuals. $I G F 2$ is monoallelically expressed in various tissues but not in the adult human liver. The human IGF2R gene, which is imprinted in mice, was found to be expressed from both alleles (KALSCHEUER et al. 1993; OGAWA $e t$ al. 1993). JINNO et al. (1994) demonstrated that WTI can undergo tissue-specific imprinting. Furthermore, they found monoallelic expression of WT1 in some placentas but not others, suggesting genetic polymorphism in imprinting within the human population.

To explain the observed patterns of genomic imprinting, DAVID HAIG and his colleagues proposed a genetic conflict hypothesis, which explains genomic imprinting as an outcome of evolution based on natural selection [HAIG and WeSTOBY 1989, 1991; HAIG and GRAHAM 1991; see VARMUZA and MANN (1994) for an alternative hypothesis ]. They noted a conflict of interest between the paternal and maternal alleles of a growth factor gene within an embryo. Consider the simplest case in which the mother mates with a large number of males, and all the offspring have different fathers. Then the paternally derived copy of a growth factor gene would try to gain as much resources as possible from the mother and thereby maximize the survivorship and growth rate of the embryo, without considering the survivorship of other embryos that have different fathers. In contrast, the maternal gene may benefit by reducing the resource demand because there is a $50 \%$ chance of copy of the gene existing in each of the other maternally related sibs. Even if the probability of a female accepting multiple mates is low, and hence most offspring are likely to have a common father, there is still some difference between the two alleles in terms of the "optimal" amount of maternal resources. HAIG (1992) demonstrated differences in the optimal amount for the maternally derived gene in the offspring, for the paternally derived gene in the offspring, and for the gene in the mother. This hypothesis explains why the paternal allele tends to be active and the maternal allele inactive for embryonic growth factor genes. In contrast, for genes coding for inhibitors of growth factors or genes that work to reduce the resource demand in general, the maternal allele tends to be active and the paternal one inactive.

In this paper, we develop a mathematical model of the evolution of genomic imprinting based on the genetic conflict hypothesis, to examine whether this idea presented as a verbal argument is supported in terms of rigorous genetic models of evolution. The model predicts the evolution of a regulatory region that controls the imprinted expression patterns for growth factor genes that affect the amount of nutrients obtained from the mother. Assuming that the "primary imprinting signal" is given, the model predicts that even with a small probability of the female accepting multiple mates, it is always very likely for the genes controlling maternal resource supply to evolve to an extreme asymmetry in gene expression. No matter how small the probability of the female accepting multiple males, the evolutionary outcome is always an extreme asymmetry in gene expression between maternally and paternally derived genes. This result, however, does not apparently fit with the observed patterns in that not all the genes affecting nutrient demand are imprinted and some genes are imprinted in mice but not in humans.

We then discuss several modified versions of the model, incorporating processes that favor the absence of genomic imprinting. In particular we study the possibility that recessive deleterious mutations may be present in the coding region of the gene. We demonstrate that whether genomic imprinting should evolve is affected by the frequency of deleterious mutations. Two equilibria, one with genomic imprinting and the other without imprinting, can be simultaneously stable.

\section{THE MODEL}

We consider the level of expression of a gene coding for an embryonic growth factor that determines the demand for maternal resources obtained through the placenta. An embryo has a pair of genes: one from the father and the other from the mother. Let $x$ and $y$ be quantitative traits indicating the amount of a growth factor produced by the paternal gene and by the maternal gene, respectively. These are either positive or zero, as they indicate gene expression levels. We assume that their sum, $x+y$, is proportional to the amount of resources or care received from the mother. Here, we 


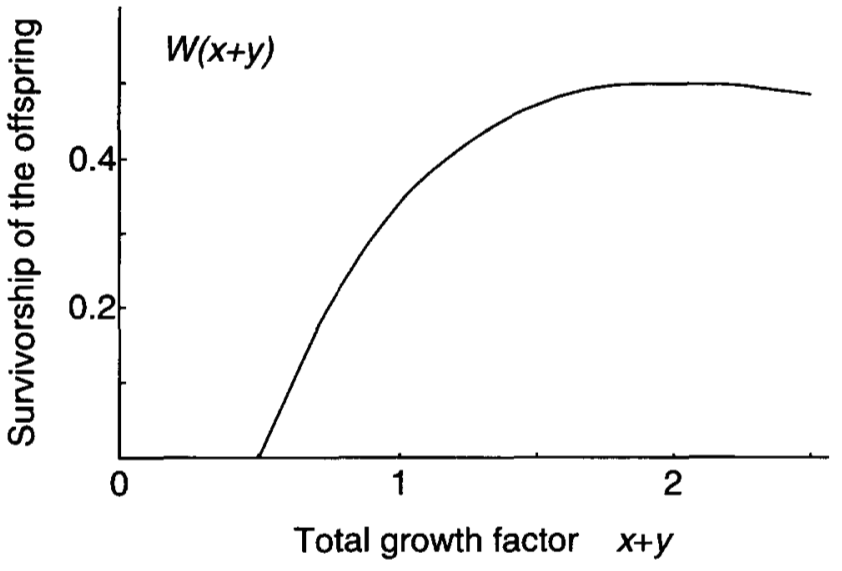

FIGURE 1.-Survivorship of an embryo as a function of the amount of growth factor production $z=x+y \cdot W(z)=w_{0}(z$ $-\alpha) /\left(1+\beta z^{2}\right)$ for $z \geq \alpha$, but is zero for $z<\alpha$. Parameters are as follows: $\alpha=0.5, \beta=0.5$ and $w_{0}=1$. This is a function used for computation in all the numerical results in this paper, unless otherwise stated. Survivorship peaks when $z=2$. Since an offspring with growth factor production lower than $\alpha$ dies, only evolution for region $z \geq \alpha$ is considered.

examine the evolutionary change in $x$ and $y$, in particular, the potential evolution toward asymmetric expression from an initial symmetry. We treat the physiological response of the mother to the growth factor as given, and do not consider the evolution of the mother's response itself.

The survivorship of the embryo increases with maternal resource supply, and hence it is a function of $x+$ $y$, denoted by $W(x+y)$. An example of a survivorship function is illustrated in Figure 1. It is zero (or very small) below a certain threshold then increases quickly with $x+y$, and finally saturates at a high levels. There may also be a peak after which it may decline with $x+$ $y$ due to any harmful effects of producing too much growth factor.

This curve gives the survivorship of the offspring as a function of the amount of resources it receives from the mother. The embryonic growth factor production $(x+y)$ that maximizes survivorship is however not optimal, because the embryo shares the common pool of maternal resources with its sibs, either simultaneously or over the mother's reproductive lifetime. Hence reducing its resource demand will increase the number and the survivorship of sibs that are genetically related to the embryo. The level of growth factor production favored by natural selection should therefore be lower than the level that achieves the maximum survivorship of the embryo. The optimal growth factor production for the maternally derived gene is still lower than that for the paternally derived gene, because the former is more likely to be present in sibs than the latter, if the mother has accepted multiple males. Therefore, there is a conflict of interest between the alleles even though they are both present in the same cells of the embryo.

The primary imprinting mechanism is still under in- vestigation, but it is suspected that differential methylation during gametogenesis plays a role (CHAILLET et al. 1991; SASAKI et al. 1991; UEDA et al. 1992; Li et al. 1993). Here we simply assume that some mechanisms exist that give a reliable primary signal that indicates parental origin, and we concentrate on the evolution of the quantitative level of expression of a growth factor gene with this signal.

We assume that a gene can produce the growth factor at two different levels in each generation, depending on its parental origin. This property of the growth factor gene is represented as a pair of values $(x, y)$, where $x$ is the amount of growth factor produced by the gene if it is paternally transmitted (i.e., given the paternal signal), and $y$ is the production by the same gene if it is maternally transmitted. In a single fertilized egg, there are two copies (paternal and maternal), each of which has a pair of values. Let $\left(x_{p}, y_{p}\right)$ be the paternally derived allele, and $\left(x_{m}, y_{m}\right)$ be the maternally derived allele. By definition, the former produces $x_{p}$ and the latter produces $y_{m}$, and hence the embryo should produce $x_{p}+y_{m}$ growth factor in total, which is proportional to the amount of resources acquired through the placenta. The survivorship of the embryo is then $W\left(x_{p}\right.$ $\left.+y_{m}\right)$.

Now we consider the fitness of the $(x, y)$ allele, defined as the expected number of copies in the following generation for each gene. We calculate the fitness as the multiplication rate from the adult stage in a generation to the adult stage in the next generation (not from the embryo to the embryo, as explained below). It depends on the sex of the gene carrier. Let $\phi_{m}(x, y ; \bar{x}, \bar{y})$ be the fitness for an $(x, y)$-allele in a male. It therefore depends on the population mean traits $(\bar{x}, \bar{y})$. Similarly, we consider $\phi_{f}(x, y ; \bar{x}, \bar{y})$, as the fitness of the $(x, y)$ allele in a female's body. We call $\phi_{m}$ and $\phi_{f}$ male and female fitness, respectively, of the allele $(x, y)$.

In the initial population, the two values may be equal $(\bar{x}=\bar{y})$, indicating the absence of genomic imprinting. In time, natural selection working on the regulatory region may cause some difference in the gene expression between the paternal and maternal copies. We model this process using multi-variate quantitative genetics. The heritable values of $x$ and $y$ of individuals in the population are assumed to be sharply concentrated around their means $(\bar{x}$ and $\bar{y})$. One-generation changes in the population averages of two quantitative traits are given by the product of a genetic variance matrix and a selection gradient vector (see IWASA et al. 1991):

$$
\left(\begin{array}{c}
\Delta \bar{x} \\
\Delta \bar{y}
\end{array}\right)=\left(\begin{array}{cc}
G_{x} & B \\
B & G_{y}
\end{array}\right)\left(\begin{array}{l}
\beta_{x} \\
\beta_{y}
\end{array}\right) .
$$

$G_{x}$ and $G_{y}$ are additive genetic variances for $x$ and $y$, respectively, and $B$ is the additive genetic covariance between them. These are determined by the balance of several different forces, such as mutation and stabilizing 
selection, pleiotropy and assortative mating, but here we simply treat them as constant, and assume that the genetic variance-covariance matrix is not degenerate (having an inverse). This is acceptable if we concentrate on the evolutionary equilibrium rather than the transient. $\beta_{x}$ and $\beta_{y}$ are selection gradients with respect to the expression of maternally derived and paternally derived genes in the embryo:

$$
\begin{aligned}
& \beta_{x}=\frac{\partial}{\partial x} \frac{1}{2}\left(\ln \phi_{m}+\ln \phi_{f}\right) \text { and } \\
& \beta_{y}=\frac{\partial}{\partial y} \frac{1}{2}\left(\ln \phi_{m}+\ln \phi_{f}\right),
\end{aligned}
$$

where the partial derivative is estimated at the population averages $((x, y) \rightarrow(\bar{x}, \bar{y}))$. These indicate the direction and magnitude of natural selection working on $x$ and $y$, respectively. The factor $1 / 2$ indicates sexlimitation, i.e., the allele is a paternally derived gene in half of all the generations, and a maternally derived gene in the other half. Justification of the evolutionary dynamics Equation 1, a and b, is given by IWASA et al. (1991). The assumption of weak selection is used for the derivation. The formulation of Equation 1 has been used in computing evolutionary equilibrium and evolutionary limit cycle for male sexual ornaments and female mating preferences (e.g., POMIANKOWSKI and IWASA 1993; IWASA and POMIANkowski 1995).

In Equation 1, $\mathrm{a}$ and $\mathrm{b}$, the effect of social interactions (in this case competition for maternal resources between sibs) is incorporated in computing the fitness, and defined as the expected number of copies in the following generation. This is called the "neighbor modulated fitness" method (HAmilton 1964). Alternatively, we may consider the cost and benefit of an embryonic gene producing different amounts of growth factor, by separating the direct effect on its own survivorship and the indirect effect on the number and survivorship of its sibs, weighted by relatedness (HAMILTON 1964). The latter approach is based on inclusive fitness, and is very useful because it gives a clear intuitive understanding of the evolutionary stability. However the inclusive fitness approach is justified only for the invadability of rare mutants to the equilibrium, and not for calculating continual change in quantitative characters. In this paper we need to trace the evolutionary trajectory of two quantitative traits and hence we adopt the straightforward quantitative genetic formulation Equation 1.

To specify the dynamics in Equation 1, we need to compute the fitness functions $\phi_{m}(x, y ; \bar{x}, \bar{y})$ and $\phi_{f}(x$, $y ; \bar{x}, \bar{y})$ from the survivorship function of the offspring $W(z)$. In this step we must specify how embryos of the same mother compete for maternal resources and how they are related genetically with each other. In the following, we examine a model in which the mother produces a large number of offspring simultaneously, the offspring share the resources, the total amount of which is limited.

We consider the fitness of a single gene of type $(x$, $y$ ) in a reproductive female's body. The expected number of copies in the next generation is the expected number of offspring produced by the female, multiplied by the probability that the copy of the allele is transmitted to each offspring, and also by the survivorship of the embryo carrying the allele. The survivorship depends on the alternative gene from her mate in the embryo. We adopt two assumptions. First, a reproductive female has a limited amount of resources $T$ that can be used by her offspring. The amount of resource allocated to each embryo is proportional to the growth factor produced by the embryo with a proportionality coefficient $a$. Hence the total number of offspring is $T$ divided by the average resource demand per embryo. Second, the female may mate with a single male or with two males, the latter occurring with probability $g$, which we call the female polygamy rate. When a female mates with two mates, the males are equally likely to sire her offspring. Under these assumptions, we calculate the fitness of an allele $(x, y)$ in a population in which breeding values are concentrated sharply around $(\bar{x}, \bar{y})$. According to the calculations in APPENDIX A, the fitness of allele $(x, y)$ in a female is

$$
\phi_{f}(x, y ; \bar{x}, \bar{y})=\frac{T}{a\left(\bar{x}+\frac{y+\bar{y}}{2}\right)} \frac{1}{2} W(\bar{x}+y) .
$$

This can be interpreted intuitively as the product of three factors: the first factor of the RHS is the expected number of offspring, which is the total resource $T$ divided by the average amount of resource demand per offspring. The average resource demand per offspring is calculated from the growth factor production by the allele concerned $(y)$, the average production by alternative alleles in the female $(\bar{y})$, and the average production by the allele from her mate $(\bar{x})$. The second factor $1 / 2$ is the probability that the allele concerned will be transmitted to each offspring. The third factor is the survivorship of an embryo, which is a function of the total growth factor production, $\bar{x}+y$, the sum of the products of paternal and maternal alleles. From the assumption of random mating, there is no correlation between the genetic values of the allele from the female and that from her mate. Absence of inbreeding also assures that the two homologous alleles in the same female are not correlated. For the purpose of evaluating the selection gradients in Equation 1, i.e., the partial derivative of fitness with respect to the breeding values of the alleles of concern ( $x$ or $y$ ), we can set all the other breeding values to population averages (see APPENDIX A, for details). Note that female fitness Equation 2a is independent of the female polygamy rate $g$.

Next, we consider $\phi_{m}(x, y ; \bar{x}, \bar{y})$, the male fitness of 
an $(x, y)$-allele in the population with mean traits $(\bar{x}$, $\bar{y})$. Let $M$ be the expected mean number of females mating with the male. $M$ may depend on the population size, the number of males and other variables, but it is assumed independent of $x$ or $y$. The probability of an allele in a male being transmitted to each embryo depends on the number of males that a female accepts. If the female accepts a single male (with probability ( 1 $-g)$ ), the probability is $1 / 2$, but if the female accepts two males [with probability $g$ (see APPENDIX A for the reason of another factor 2 ) ], the probability is $1 / 4$. The average resource demand per embryo also depends on the number of males that a female accepts. If the female accepts a single male, the average resource demand is $\bar{y}+(x+\bar{x}) / 2$, but if the female accepts two males, it is $\bar{y}+(x+3 \bar{x}) / 4$. The survivorship of the embryo carrying the $(x, y)$-allele is $W(x+\bar{y})$. We can derive the fitness of the allele $(x, y)$ in a male as

$$
\phi_{m}(x, y ; \bar{x}, \bar{y})
$$

$$
\begin{array}{r}
=M \frac{T}{a}\left[\frac{(1-g)^{1 / 2}}{\bar{y}+\frac{x+\bar{x}}{2}}+\frac{2 g^{1 / 4}}{\left.\bar{y}+\frac{x+3 \bar{x}}{4}\right]}\right. \\
\times W(x+\bar{y}) .
\end{array}
$$

In APPENDIX A, we also derived the fitnesses for the case in which females may accept more than two males.

Substituting Equation 1 by Equations $2 \mathrm{a}$ and $2 \mathrm{~b}$ gives us the evolutionary dynamics of the average traits $(\bar{x}$, $\bar{y})$. Figure 2 illustrates a typical trajectory of the evolutionary dynamics of $(\bar{x}, \bar{y})$. Starting from any initial state the population quickly converges to a line of $x+$ $y=$ constant and then moves toward a biased expression with $\bar{x}$ increasing and $\bar{y}$ decreasing, finally converging to the state in which only the paternal allele is expressed $(\bar{x}>0, \bar{y}=0)$, thus implying extreme imprinting of the growth factor gene. When the population reaches this state, the mean trait should stop decreasing further, since a negative value of $\bar{x}$ or $\bar{y}$ is not biologically meaningful.

This behavior can be understood as follows: Suppose that the total growth factor production optimal for the paternal allele $\bar{x}+\bar{y}=z_{m}$ is a little larger than that optimal for the maternal allele $\bar{x}+\bar{y}=z_{f}\left(z_{m}>z_{f}\right)$, two parallel lines on the $(\bar{x}, \bar{y})$-plane are formed. The initial transient is the quick convergence of the average traits $(\bar{x}, \bar{y})$ to the region between these two lines, in which the total growth factor production is smaller than the paternal allele optimum but larger than the maternal allele optimum. Subsequently $\bar{x}$ increases and $\bar{y}$ decreases very slowly but the population average traits $(\bar{x}$, $\bar{y})$ stay in the region between two lines. The asymmetry in gene expression increases and the final outcome is on the boundary of the positive orthant, where only the

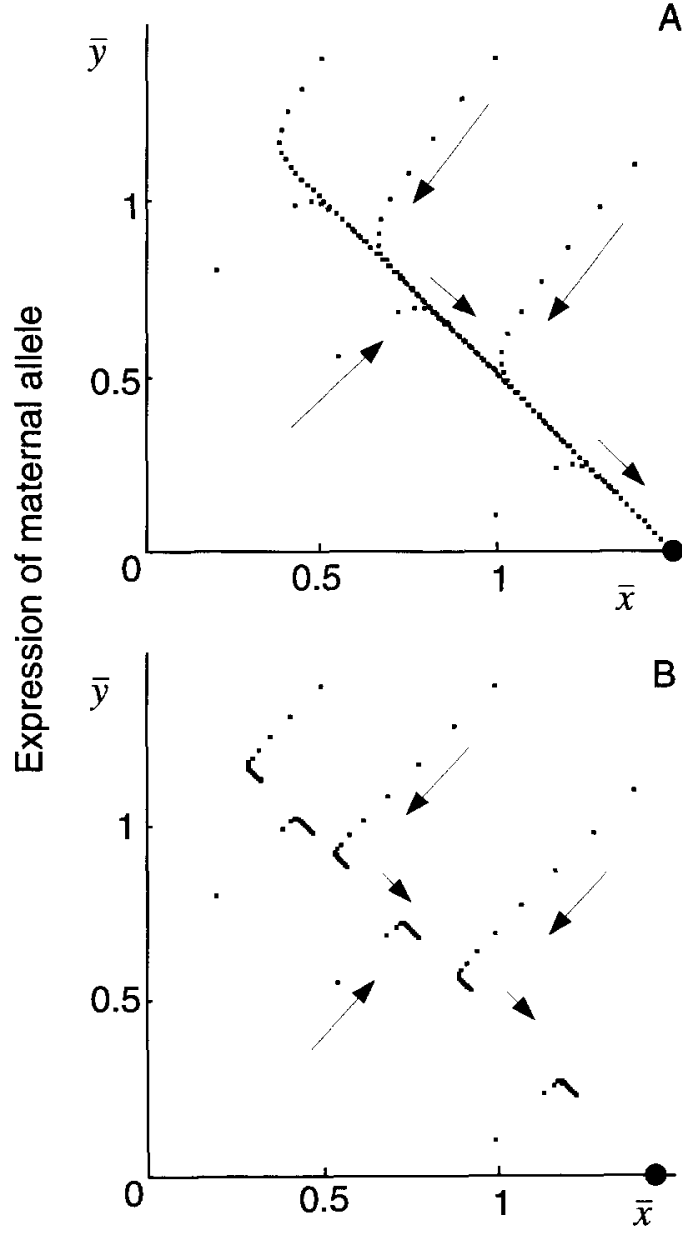

\section{Expression of paternal allele}

FIGURE 2.-Evolutionary trajectories of the basic model. The two axes are population average of paternal allele expression $\bar{x}$ and maternal allele expression $\bar{y}$. (A) Female polygamy rate is large $(g=0.5)$. Other parameters are $G_{x}=G_{y}=$ $0.2, B=0$, and $W(z)$ given by Figure 1 . (The dynamics are independent of $T, A$, or $M)$. The location of $(\bar{x}, \bar{y})$ for every other generation is indicated. (B) Parameters are the same as in A except female polygamy rate is small $(g=0.05)$. There is a globally stable equilibrium on the $\bar{x}$-axis in which the maternal allele is silent. This implies that strong genomic imprinting should evolve and that only the paternal copy of the gene is expressed. The speed of the convergence to the final equilibrium depends on the female polygamy rate $g$.

paternal allele is expressed and the maternal allele is silent.

In APPENDIX A, we show that this conclusion is quite general: there is no equilibrium satisfying $\bar{x}>0$ and $\bar{y}$ $>0$, provided that there is some probability of a female accepting multiple males $(g>0)$. When $g=0$, the two lines where there are no selection gradients $\left(\beta_{x}=\beta_{y}\right.$ $=0$ ) coincide exactly with each other, producing a line of equilibria.

If there is only a small probability of females accepting multiple mates, we might expect that an intermediate degree of imprinting could evolve, so that two alleles are expressed at different levels. The present 
model does not support this conjecture. There is no possibility of a positive equilibrium in which both the paternal and maternal genes are expressed. If the degree of female polygamy $g$ is small, the evolutionary movement toward genomic imprinting is slow, but even a slight possibility of multiple mating by the female is enough to make the evolution of genomic imprinting a certainty (Figure 2). Complete monogamy, $g=0$, is found rarely if at all in nature with the study of the mating systems of mammals revealing that some degree of sperm competition is common (DewsBury 1984; HarVey and Harcourt 1984; SMith 1984). We can therefore conclude that the model predicts that genomic imprinting evolves easily in genes that affect the embryo's growth.

However, contrary to this prediction, there are growth factors that affect the formation of the placenta that are not imprinted. For example, the $\operatorname{Ig} f 1$ gene is imprinted in neither mice nor humans despite its marked effect on the development of the embryo (LIU et al. 1993). In addition, $\operatorname{Ig} f 2 r$ in the placenta, which is a suspected inhibitor of $\lg f 2$ (HAIG and GRAHAM 1991 ), is maternally imprinted in the mouse but not in humans (KALSCHEUER et al. 1993; OGAWA et al. 1993). These findings are in conflict with the simple and general prediction of the model that all the genes affecting the amount of nutrients from the mother should evolve strong differential expression. Hence there must be some additional processes that inhibit the evolution of strong genomic imprinting.

\section{EFFECT OF DELETERIOUS MUTATIONS}

These discrepancies can be explained by additional processes that favor equal expression of both genes, thereby discouraging the evolution of genomic imprinting. Here we examine the most plausible candidate; the presence of deleterious mutation in the coding region of the gene.

The point is that there is a mutation-selection balance at all loci that creates a selection against imprinting. Intuitively speaking, the advantage of not being imprinted, i.e., having biallelic expression, is the same as the advantage of being diploid. Since recurrent recessive deleterious mutations are thought to be the most important process favoring diploidy (e.g., PERROT et al. 1991 ), they are a promising candidate to explain the disadvantage of genomic imprinting. Hence, we consider deleterious mutations in the structural gene of the growth factor that may affect the evolution of the regulatory region. In the population, such deleterious nonfunctional mutations occur every generation and are eliminated by natural selection. They are maintained at a frequency determined by the balance between mutation and selection. We assume that linkage between the regulatory region and the structural gene is tight, and we consider only the regulatory region linked with a wild-type (functional) structural gene. Regulatory regions linked with a defective structural gene are neglected, since they will be eliminated from the population. However, the evolution of a regulatory region linked with a wild-type gene can still be affected by the existence of mutations in the population because they may be associated within the same embryo.

Let $f$ be the frequency of deleterious (or null) mutation of the structural gene at equilibrium. Although $f$ may change in evolution, here we treat $f$ as a constant for the sake of clarity of argument. If the gene is imprinted in the population $(x>0$ and $y=0)$, paternal deleterious mutants produce a phenotype deficient in growth factor, though the maternal deleterious mutant is normal. In contrast, if the gene is not imprinted ( $x$ $=y$ ), deleterious mutations (both maternal and paternal) produce a phenotype with growth factor production at a lower rate (half of the normal phenotype). Considering the homeostatic adjustment of development, reducing the amount of growth factor would be less serious to embryonic development than the complete absence of growth factor, which is likely to be lethal. The possibility of being associated with deleterious genes may discourage the evolution of genomic imprinting. This conjecture may, however, depend on the shape of survivorship as a function of growth factor production $W(z)$ and needs to be examined using quantitative mathematical models.

In the model, a female may mate with a single male or two males as her mates, with probability $1-g$ and $g$, respectively. In computing the fitness of an $(x, y)$ allele in a reproductive female, we need to separate cases according to whether she and her mate (s) carry a mutant or a wild-type copy. Since the mutant homozygotes are lethal $(W(0)=0)$, the adult population is composed of wild-type homozygotes $(+/+)$ and heterozygotes $(+/-)$ only. We denote the frequency of the mutant gene in the population by $f$. Then the fraction of wild-type homozygotes $(+/+)$ is $1-2 f$ and that of heterozygotes $(+/-)$ is $2 f$, for both males and females. A random mating assumption allows us to use these fractions in computing the combination of the genotypes of a female's mates. On the other hand, the female herself is either heterozygous or homozygous, with probability $(1-2 f) /(1-f)$ and $f /(1-f)$, respectively (further explanation is in APPENDIX B). By computing the fitness of the $(x, y)$-allele for different cases and by averaging them with the probability of these cases, we have the female fitness function $\phi_{f}$. A male fitness function $\phi_{m}$, the expected number of copies of the $(x, y)$-allele linked with a wild-type structural gene in males is also derived in APPENDIX $\mathbf{B}$. The results are straightforward but are too bulky to show in the text. By replacing these in Equation 1, we have the evolutionary dynamics of $(\bar{x}, \bar{y})$.

The evolutionary outcome is as follows: (1) no imprinting when $f$ is large, or (2) full imprinting when 


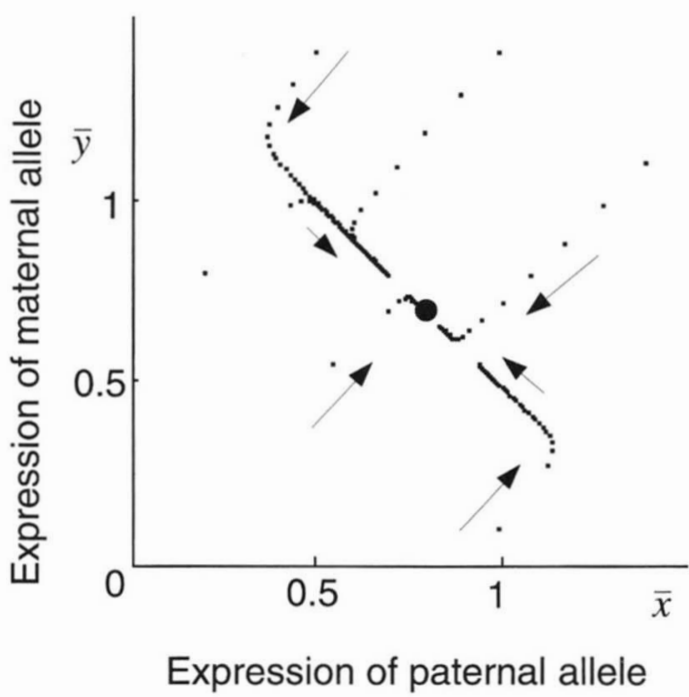

FIGURE 3.-Evolutionary trajectories with deleterious recessive mutations of the structural gene. Frequency of deleterious mutations is $f=0.02$. Other parameters are the same as in Figure 2B. At the evolutionary equilibrium, both paternal and maternal alleles are expressed $(\bar{x}>0, \bar{y}>0)$, implying the absence of strong genomic imprinting.

$f$ is small, or (3) an intermediate degree of imprinting when $f$ is intermediate. Figure 3 shows the evolutionary trajectories of the model in case 1 . Even for polygamy rate $g>0$, if $f$ is significantly large, there is a stable equilibrium in the middle of the positive orthant $(\bar{x}>0$ and $\bar{y}>0)$. In this particular case, maternal and paternal genes are expressed at a similar level, indicating the absence of genomic imprinting.

A large polygamy rate $g$ causes a strong conflict of interest between two growth factor alleles with different parental origin and results in a strong asymmetry in the expression levels. On the other hand, genomic imprinting is discouraged by deleterious mutations with frequency $f$ in the population. In Figure 4 , the relative magnitude of production of maternal alleles to that of paternal alleles $(\bar{y} / \bar{x})$ is illustrated as a contour map over two parameters: the frequency of deleterious mutations $f$ and female polygamy rate $g$. The basic model discussed in the previous section corresponds to the points on the vertical axis $(f=0)$. In the shaded region near the vertical axis, strong genomic imprinting should evolve $(\bar{y}=0)$. As the frequency of mutants in the population $f$ increases and exceeds a line on this plane, the maternal alleles becomes expressed, though at a level lower than the paternal allele. As the ratio of $f$ to $g$ increases, the difference between maternal and paternal alleles becomes smaller, reducing the degree of genomic imprinting.

\section{DISCUSSION}

We examined mathematical models of the evolution of differential expression of the paternally and the maternally derived alleles of growth factor genes, genomic

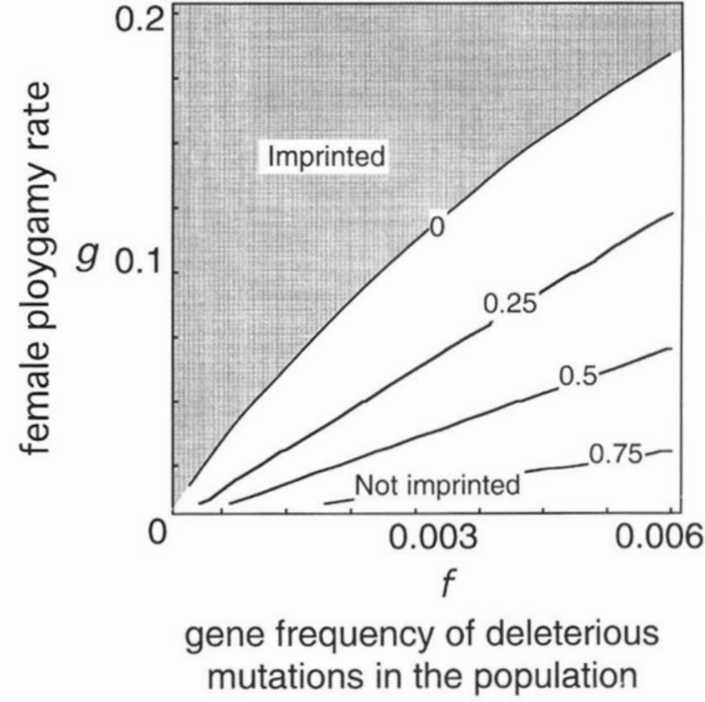

FIGURE 4.-The ratio of the expression of the maternal allele to the paternal allele at evolutionary equilibrium $\bar{y} / \bar{x}$. Horizontal axis is the frequency of deleterious mutations in the population $f$, vertical axis, female polygamy rate $g$. The resource division model with survivorship curve in Figure 1 is assumed.

imprinting, by considering the cis-acting regulatory region controlling the level of expression dependent on parental origin. We observed that asymmetric expression of the two alleles evolves very easily from the initial symmetry, if there is even a small chance of a female mating with more than one male. By slight modification, we can show that the gene coding for inhibitors should evolve genomic imprinting in the reverse direction with the paternal allele becoming silent, as explained in APPENDIX C. This confirms the genetic conflict hypothesis, previously stated verbally by DAVID HAIG and his colleagues (HAIG and Westoby 1989, 1991; Haig and Graham 1991; Moore and Haig 1991 ). What is notable in the basic model is that the evolutionary outcome is always an extreme asymmetry even if the probability of female polygamy is small.

In this paper, we examine the case of a "resource division model" in which the mother produces a large number of offspring simultaneously and the offspring share the resources. In many mammals, however, a single offspring is gestated at a time, and the production of the next offspring becomes possible only when the mother stops investing in the "current" offspring, which is apparently in conflict with the assumptions of the resource division model. In a separate paper, we examine the "sequential care model" in which offspring are born and receive maternal care one at a time (Y. IWASA, A. MOCHIZUKI and Y. TAKEDA, unpublished results). The survivorship of an offspring increases with the length of time that the mother invests her care to that offspring. A longer period of care for each individual reduces the total number of offspring produced in the mother's lifetime. The result of this model is 
qualitatively the same as the present model. If the probability that a female accepts multiple mates is positive, the expression level of the gene of a growth factor should evolve to show extreme asymmetry. We will show this result in another paper.

The ease of the evolution of genomic imprinting predicted by the basic model is not totally consistent with the observation. Some genes known to control the embryonic development are not imprinted (LIU et al. 1993 ), and there are genes that are imprinted in the mouse but not in humans (KALSCHEUER et al. 1993; OGAWA et al. 1993).

To explain why there are only a few genes imprinted in mammals, we considered models that incorporated potential processes that favor equal expression of the maternal and paternal alleles in the embryo. We examined one candidate, the effect of deleterious mutation in the coding region of the growth factor gene. Depending on the values of the polygamy rate and the frequency of mutation in the population, the model predicts either the absence or the presence of genomic imprinting.

In a separate paper (Y. IWASA, A. MochIZUKI and Y. TAKEDA, unpublished results), we examined two other candidate processes that potentially favor the absence of genomic imprinting. First, a mistake in labeling the parental origin of one allele would make both genes in an embryo stay silent or both genes be active, resulting in deficient or overproduction of growth factor. Hence it is possible that this may disfavor the evolution of genomic imprinting. However, mistakes occur too infrequently (REIK et al. 1995) to be important. Second, the possibility of differential expression causing additional energy and time expenditure that may reduce the fitness by a small amount therefore favors the absence of genomic imprinting. The models considered are qualitatively the same as the model with the deleterious mutations examined in this paper. Depending on parameter values, the models predict genomic imprinting may or may not evolve.

The analysis in this paper suggests that whether an embryonic growth factor gene evolves to become imprinted or not may be determined by the balance between the polygamy rate and the frequency of deleterious mutations of the structural gene in the population. Imprinting is more likely to evolve in a population with a high polygamy rate. Since the polygamy rate is the same for different genes of the same species, the variation between genes in terms of imprinting should be explained by the difference in the frequency of deleterious mutations per structural gene, the latter determined by the mutation-selection balance. Hence a gene including more of functional sites should have a higher mutant rate per gene and is less likely to be imprinted than genes with fewer functional sites.

Recent study of the molecular mechanism of imprinting has revealed that imprinted genes tend to form clusters that are located in "imprinted chromosomal domains", and that many genes tend to be controlled by a single "imprinting control element" (REIs et al. 1994; SUTClifFe et al. 1994; BUITING et al. 1995; EDEN and CEDAR 1995). This suggests that reliable information on the parental origin of a chromosome is not always available, and once it is available then many genes may in fact evolve to show genomic imprinting. If this interpretation is correct, the conclusion of the basic model in the present paper may be justified. The question then is why are imprinted chromosomal regions rare. The hypothesis of the cost of imprinting including all the molecular machinery for imprinting may be worth more careful examination.

In this paper, in examining the evolutionary change in the level of gene expression, we assumed that a primary signal for parental origin is given. Underlying this is the assumption that the degree of expression should be determined by the nucleotide sequence of the regulatory region of the growth factor genes. The primary imprinting of the gene should be given during the gametogenesis. If the pattern of imprinting for a gene is determined by the parental molecular machinery rather than the DNA sequence that is to become imprinted, we need to examine a model in which the parental diploid genotype would determine the pattern of imprinting for their gametes. The predicted evolutionary outcome should be different from the analysis in the present paper due to parent-offspring conflict (TrIvers 1974; Queller 1994). Another form of involvement of the parental genome is the reaction of the mother to the amount of growth factor produced in each embryo, which is certainly an important theme of future theoretical investigation (see HAIG 1993). In the current paper we concentrate on the embryo's growth factor production by simply assuming that the mother would invest more to embryos producing more growth factor.

We considered the evolution of cis-acting regulatory regions of growth factor genes and succeeded in explaining patterns of extreme asymmetry of gene expression depending on the parental origin. This in effect explains genomic imprinting as "voluntary" regulation of maternally derived and paternally derived alleles. MoORE et al. (1995) and MoORE and REIK (1996) suggested additional mechanisms by which an allele suppresses the opponent allele directly: Some substances in the oocyte cytoplasm may modify the paternally derived allele after fertilization to control the expression level of the allele, which can be counteracted by the sperm through the production of cytosolic factors. They also suggest that such postzygotic reprogramming may be a reason for the nonimprinting of genes that affect the growth of the embryo.

If a different survivorship function $W(z)$ is adopted, some of the conclusions can be changed for the case with deleterious mutations favoring the absence of im- 

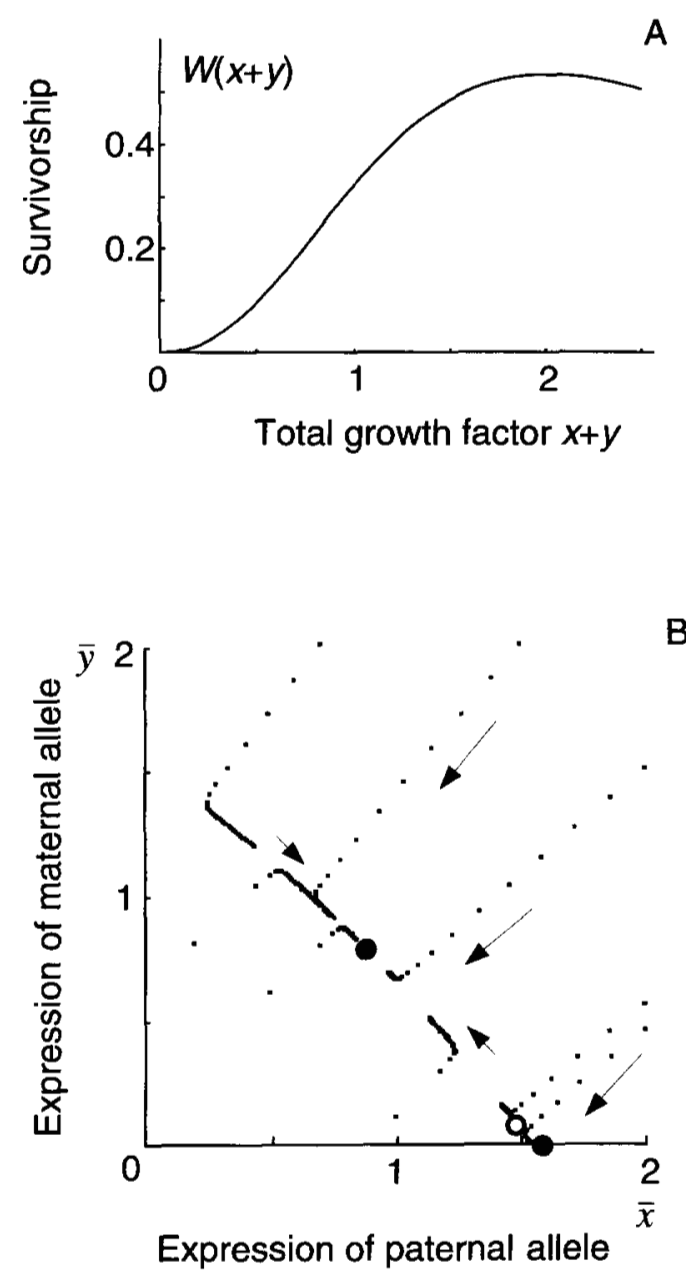

FigURE 5.-(A) Survivorship of an embryo as a function of the amount of growth factor production, $W(z)=w_{0} z^{2} /(1+$ $h z^{3}$ ), with $w_{0}=0.4, h=0.25$. This is slightly different from the one in Figure 1. (B) Evolutionary trajectory with deleterious recessive mutation of structural genes (resource division model). Parameters are as follows: $g=0.1, f=0.08$. There are three equilibria, one stable equilibrium without genomic imprinting $(\bar{x}>0, \bar{y}>0)$, another stable equilibrium with genomic imprinting $(\bar{x}>0, \bar{y}=0)$, and an unstable equilibrium lying between these two. The system is bistable, and the evolutionary outcome depends on the initial population.

printing. For example, Figure 5B illustrates the evolutionary trajectories when the survivorship function is given by Figure 5A, which is slightly different from the one in Figure 1 used to generate Figures 2 and 3. For a particular parameter values of $g$ and $f$, we obtain the evolutionary trajectories shown in Figure $5 \mathrm{~B}$, in which the system is bistable, having three equilibria, two of which are locally stable. One stable equilibrium is in the middle of the graph, indicating that both paternal and maternal alleles are expressed. The second stable equilibrium is on the $\bar{x}$-axis indicating that the maternally derived allele is inactive and that the paternally derived allele is strongly expressed. Between these two stable equilibria, there is an unstable equilibrium. Evolutionary trajectories should be separated into two domains of attraction, each corresponding to one of the two stable equilibria. Hence the model predicts that, depending on evolutionary history, two species having similar ecology and mating system may show extremely different levels of genomic imprinting. This may explain the difference in imprinting pattern between mice and humans (KALSCHEUER $e t$ al. 1993; OGAWA $e t$ $a l$. 1993). However the range of parameter values $f$ and $g$ that allow the evolution of bistability as shown in Figure $5 \mathrm{~B}$ is quite small.

In addition to the genetic conflict hypothesis, several alternative hypotheses have been proposed to explain the pattern of genomic imprinting. For example, VARMUZA and MANN (1994) proposed that imprinting might be a device that protects female mammals from the potential ravages of ovarian trophoblast disease caused by the spontaneous start of the development of unfertilized eggs. If the maternal allele is silent $(\bar{y}=$ 0 ), the risk of ovarian trophoblast disease would be reduced, to the advantage of both parents, thus favoring the reduction of maternal gene expression. This, together with subsequent compensatory evolution of paternal gene expression, would produce the evolution of genomic imprinting, even in the absence of a conflict of interest between paternal and maternal alleles. This hypothesis can be studied by a similar theoretical framework to the present paper, but we would like to pursue this project in a separate paper.

To examine the feasibility of several different hypotheses proposed to explain the phenomena of genomic imprinting, to establish when and how each mechanism works, and to predict quantitatively the relative importance of the alternative hypotheses, we need to develop theoretical studies for each, just as we have in this paper for the genetic conflict hypothesis.

We express our sincere thanks to Professor HiRovuki Sasaki, Research Laboratory for Genetic Information, Kyushu University, who kindly introduced us to the problem of genomic imprinting. We also thank following people for their very useful comments: M. BOOTS, C. Godfray, D. Haig, I. Kobayashi, Hirotsuga Matsuda, Hiroyuki Matsuda, T. Moore, W. Reik, A. Sasaki, A. Surani, K. Takahashi, S. Takahashi, M. Uyenoyama and T. Yahara. This work was supported in part by a Grant-in-Aid for Scientific Research by the Ministry of Education, Science, and Culture, Japan.

\section{LITERATURE CITED}

Barlow, D. P., R. Stogfr, B. G. Herrmann, K. Saito and N. SCHWEIFER, 1991 The mouse insulin-like growth factor type-2 receptor is imprinted and closely linked to the Tme locus. Nature 349: $84-87$.

Bartolomei, M. S., S. Zemel and S. M. Tilghman, 1991 Parental imprinting of the mouse H19 gene. Nature 351: 153-155.

Buiting, K, S. Saitoh, S. Gross, B. Dittrich, S. Schwartz et al, 1995 Inherited microdeletions in the Angelman and PraderWilli syndromes define an imprinting centre on human chromosome 15. Nature Genet. 9: 395-400.

Chaillet, J. R., T. F. Vogt, D. R. Beier and P. Leder, 1991 Parentspecific methylation of an imprinted transgene is established during gametogenesis and progressively changes during embryogenesis. Cell 66: $77-83$.

DeGhiara, T. M., E. J. Robertson and A. Efstratiadis, 1991 Parental imprinting of the mouse insulin-like growth factor II gene. Cell 64: 849-859. 
DEwSBURY, D. A., 1984 Sperm competition in muroid rodents, pp. 547-571 in Sperm Competition and the Evolution of Animal Mating Systems, edited by R. L. SMITH. Academic Press Inc., New York.

EDEN, S., and H. CEDAR, 1995 Action at a distance. Nature 375: 1617.

Ferguson-Smith, A. C., B. M. Cattanach, S. C. Barton, C. V. BEECHEY and M. A. SURANI, 1991 Embryological and molecular investigations of parental imprinting on mouse chromosome 7. Nature 351: $667-670$

HaIG, D., 1992 Genomic imprinting and the theory of parent-offspring conflict. Sem. Dev. Biol. 3: 153-160.

HAIG, D., 1993 Genetic conflicts in human pregnancy. Q. Rev. Biol. 68: $495-532$

HAIG, D., and C. GRAHAM, 1991 Genomic imprinting and the strange case of the insulin-like growth factor II receptor. Cell 64: $1045-1046$

HAIG, D., and M. Westoby, 1989 Parent-specific gene expression and the triploid endosperm. Am. Nat. 134: 147-155.

HAIG, D., and M. WEstoby, 1991 Genomic imprinting in endosperm: its effect on seed development in crosses between species, and between species, and between different ploidies of the same species, and its implications of the evolution of apomixis. Phil. Trans. R. Soc. Lond. B 133: 1-13.

HAMILTON, W. D., 1964 The genetical evolution of social behaviour. J. Theor. Biol. 7: 1-52.

Hao, Y., T. Crenshaw, T. Moulton, E. Newcomb and B. Tycko, 1993 Tumour-suppressor activity of H19 RNA. Nature 365: 764-767.

HaRVEY, P. H., and A. H. HARCOURT, 1984 Sperm competition, testes size, and breeding systems in primates, pp. 589-600 in Sperm Competition and the Evolution of Animal Mating Systems, edited by R. L. SMith. Academic Press Inc., New York.

HATADA, I., and T. MUKAI, 1995 Genomic imprinting of $\mathrm{p}^{5} 7^{\mathrm{KIP} 2}$, a cycling-dependent kinase inhibitor, in mouse. Nature Genet. 11: 204-206.

IWASA, Y., and A. PomLANKowskI, 1995 Continual change in mate preferences. Nature 377: 420-422.

IWASA, Y, A. POMIANKOWSKI and S. NEE, 1991 The evolution of costly mate preferences. II. The 'handicap' principle. Evolution 45: $1431-1442$.

Jinno, Y., K Yun, K. Nishiwaki, T. Kubota, O. Ogawa et al., 1994 Mosaic and polymorphic imprinting of the WTl gene in humans. Nature Genet. 6: 305-309.

Kalscheule, V. M., E. C. Mariman, M. T. Schepens, H. Rehder and H.-H. Ropr.Rs, 1993 The insulin-like growth factor type-2 receptor gene is imprinted in the mouse but not in humans. Nature Genet. 5: 74-78.

LI, E., C. BEARD and R. JAENISCH, 1993 Role for DNA methylation in genomic imprinting. Nature 366: 362-365.

LiU, J.P., J. Baker, A. S. Perkins, E. J. Robertson and A. EfstratiADIS, 1993 Mice carrying null mutations of the genes encoding insulin-like growth factor $1(\operatorname{Ig} f 1)$ and type-1 IGF receptor ( Igf $1 r$ ). Cell 75: 59-72.

MOORE, T., and D. HAIG, 1991 Genomic imprinting in mammalian development: a parental genetic conflict. Trends Genet. 7: 4549.

MoOrE, T., and W. REIK, 1996 Genetic conflict in early development: parental imprinting in normal and abnormal growth. Rev. Reproduction 1: 73-77.

MoOrt, T., L. D. HuRst and W. REIK, 1995 Genetic conflict and evolution of mammalian X-chromosome inactivation. Dev. Genet. 17: 206-211.

OgaWA, O., L. A. MCMOE, R. Eccles, I. M. Morrison and A. E. REEVE, 1993 Human insulin-like growth factor type I and type II receptors are not imprinted. Hum. Mol. Genet. 2: 2163-2165.

Ozcelik, T., S. LeFF, W. ROBInSON, T. DOnlon, M. LALANDE et al., 1992 Small nuclear ribonucleoprotein polypeptide $\mathrm{N}$ ( $S N R P N$ ), an expressed gene in the Prader-Willi syndrome critical region. Nature Genet. 2: 265-269.

Peterson, K., and C. SAPIENZA, 1993 Imprinting the genome: imprinted genes, imprinting genes, and a hypothesis for their interaction. Annu. Rev. Genetics 27: 7-31.

Piass, C., H. Shlbata, I. Kalcheva, L. Mullins, N. Kotelevtseva $e$ al., 1996 RLGS-M identification of CDC25 ${ }^{\mathrm{Mm}}$ as an imprinted gene. Nature Genet. (in press).

POMLANkowskr, A., and Y. IWASA, 1993 Evolution of multiple sexual ornaments by Fisher's process of sexual selection. Proc. R. Soc. Lond. B. 253: 173-181.

Perrot, V., S. Richerd and M. Valero, 1991 Transition from haploidy to diploidy. Nature 351: 315-317.

Qufl.ter, D. C., 1994 Male-female conflict and parent-offspring conflict. Am. Nat. 144: S84-S99.

REIK, W., K. W. BROWN, H. SCHNEID, Y. LE BOUC, W. BICKMORE and E. R. MAHER, 1995 Imprinting mutations in the Beckwith-Wiedemann syndrome suggested by an altered imprinting pattern in the IGF2-H19 domain. Human Mol. Genet. 4: 2379-2385.

Reis, A., B. Dittrich, V. Greger, K. Buiting, and M. Lal.ande et al., 1994 Imprinting mutations suggested by abnormal DNA methylation patterns in familial Angelman and Prader-Willi syndromes. Am. J. Hum. Genet. 54: 741-747.

Sasaki, H., T. Hamada, T. Ueda, R. Seki, T. Higashinakagawa el al, 1991 Inherited type of allelic methylation variations in a mouse chromosome region where an integrated transgene shows methylation imprinting. Development 111: 573-581.

Sasaki, H., N. D. Allen and M. A. Surani, 1993 DNA methylation and genomic imprinting in mammals, pp. 469-486 in DNA Methylation: Molecular Biology and Biological Significance, edited by J. P. JosT and H. P. SALuz. Birkhauser Verlag, Basel.

SmITH, R. L., 1984 Human sperm competition, pp. 601-659 in Sperm Competition and the Evolution of Animal Mating Systems, edited by R. L. SMITH. Academic Press Inc., New York.

Sutci.iffe, J. S., M. NakaO, S. Christian, K. H. Orstavik, N. TomMERUP et al., 1994 Deletions of a differentially methylated CpG island at the SNRPN gene define a putative imprinting control region. Nature Genet. 8: 52-58.

Trivers, R. L., 1974 Parent-offspring conflict. Am. Zool. 14: 249264.

Ueda, T., K. Yamazaki, R. SuzukI, H. Fujimoto, H. SASAKI et al., 1992 Parental methylation patterns of a transgenic locus in adult somatic tissues are imprinted during gametogenesis. Development 116: $831-839$

VARMUZA, S., and M. MANN, 1994 Genomic imprinting-defusing the ovarian time bomb. Trends Genet. 10: 118-123.

Wevrick, R., J. A. Kerns and U. Francke, 1994 Identification of a novel paternally expressed gene in the Prader-Willi syndrome region. Human Mol. Genet. 3: 1877-1882.

Communicating editor: L. PARTRIDGE

\section{APPENDIX A}

Derivation of (2a) and (2b): Consider a gene $(x, y)$ that is possessed by a reproductive female. Let $\left(x^{\prime}, y^{\prime}\right)$ be the alternative allele in the female. She accepts a single male with probability $1-g$. Let $\left(x_{1}, y_{1}\right)$ and $\left(x_{1}^{\prime}, y_{1}^{\prime}\right)$ be the two alleles at the same locus possessed by the male. The female may accept two males with probability $g$. The two genes of the first male are $\left(x_{1}\right.$, $\left.y_{1}\right)$ and $\left(x_{1}^{\prime}, y_{1}^{\prime}\right)$, those of the second male are $\left(x_{2}, y_{2}\right)$ and $\left(x_{2}^{\prime}, y_{2}^{\prime}\right)$.

$$
\begin{aligned}
\phi_{f}= & (1-g) E\left[\frac{T}{a\left(1 / 2\left(y+y^{\prime}\right)+1 / 2\left(x_{1}+x_{1}^{\prime}\right)\right)}\right. \\
& \left.\times \frac{1}{2} \frac{W\left(y+x_{1}\right)+W\left(y+x_{1}^{\prime}\right)}{2}\right] \\
& +g E\left[\frac{T}{a\left(1 / 2\left(y+y^{\prime}\right)+1 / 4\left(x_{1}+x_{1}^{\prime}+x_{2}+x_{2}^{\prime}\right)\right)}\right.
\end{aligned}
$$




$$
\begin{gathered}
W\left(y+x_{1}\right)+W\left(y+x_{1}^{\prime}\right) \\
\left.\times \frac{1}{2} \frac{+W\left(y+x_{2}\right)+W\left(y+x_{2}^{\prime}\right)}{4}\right],
\end{gathered}
$$

where $E[\bullet]$ implies the operation of the population average with respect to $\left(x^{\prime}, y^{\prime}\right),\left(x_{1}, y_{1}\right),\left(x_{1}^{\prime}, y_{1}^{\prime}\right),\left(x_{2}\right.$, $\left.y_{2}\right)$, and $\left(x_{2}^{\prime}, y_{2}^{\prime}\right)$. Since we assume that the population is concentrated sharply around the mean values, and also that mating is random (no correlation between two homologous alleles of the same individual), then we can simply replace $x^{\prime}=x_{1}=x_{1}^{\prime}=x_{2}=x_{2}^{\prime}=\bar{x}$, and $y^{\prime}=\bar{y}$, but leave $x$ and $y$ intact, because we need to compute partial derivatives of fitness with respect to $x$ and $y$. (A1) becomes

$$
\phi_{f}=\frac{T}{a(1 / 2(y+\bar{y})+\bar{x})} \frac{1}{2} W(y+\bar{x}),
$$

which is ( $2 a)$ in the text.

In computing the male fitness, we consider a gene of type $(x, y)$ in a reproductive male and consider a female who accepted this male as her mate. Let $\left(x_{0}\right.$, $\left.y_{0}\right)$ and $\left(x_{0}^{\prime}, y_{0}^{\prime}\right)$ be the two alleles of the female.

Here we note that the probability for a randomly chosen male mates with a female that accepts two males is larger than $g$, the latter being the probability for a female to accept two males. The former probability (probability for a male to mate with a female that accepts two males) is $2 g / 1+g$, and the probability for a male to mate with a female which accepts one male is $1-g / 1+g$. Then the fitness for the male, after being multiplied by a factor $M$, is

$$
\begin{aligned}
\phi_{m}= & M \frac{1-g}{1+g} E\left[\frac{T}{a\left(1 / 2\left(y_{0}+y_{0}^{\prime}\right)+1 / 2\left(x+x^{\prime}\right)\right)}\right. \\
& \left.\times \frac{1}{2} \frac{W\left(y_{0}+x\right)+W\left(y_{0}^{\prime}+x\right)}{2}\right]+M \frac{2 g}{1+g} \\
& \times E\left[\frac{T}{a\left(1 / 2\left(y_{0}+y_{0}^{\prime}\right)+1 / 4\left(x+x^{\prime}+x_{2}+x_{2}^{\prime}\right)\right)}\right. \\
& \left.\times \frac{1}{4} \frac{W\left(y_{0}+x\right)+W\left(y_{0}^{\prime}+x\right)}{2}\right] .
\end{aligned}
$$

The denominator, $1+g$ of $2 g / 1+g$ and $1-g / 1+$ $g$ can be considered to be neglected because they are a common factor. Now by taking the average $E[\bullet]$ with respect to $\left(x^{\prime}, y^{\prime}\right),\left(x_{2}, y_{2}\right),\left(x_{2}^{\prime}, y_{2}^{\prime}\right),\left(x_{0}, y_{0}\right)$, and ( $\left.x_{0}^{\prime}, y_{0}^{\prime}\right)$ considering that the breeding values are centered around the population mean, we have $(2 b)$ in the text.

In a similar way, we can compute the case in which a female accepts $n$ males with probability $p_{n}$, which sire the offspring of the female equally. After some arithme- tics similar to that above, we have female fitness $\phi_{f}$ the same as (2a), and male fitness $\phi_{m}$ as

$$
\begin{array}{r}
\phi_{m}=\frac{M T}{a} \sum_{n=1} n p_{n}\left[\frac{\frac{1}{2 n}}{\bar{y}+\frac{x+(2 n-1) \bar{x}}{2 n}}\right] \\
\times W(\bar{y}+x) .
\end{array}
$$

With $p_{1}=1-g, p_{2}=g, p_{3}=p_{4}=\cdots=0$, (A4) becomes ( $2 b)$.

Evolutionary equilibrium: At equilibrium, the two selection gradients must be zero:

$$
\begin{aligned}
\beta_{x}=\frac{\partial}{\partial x}\left(\ln \phi_{m}\right. & \left.+\ln \phi_{f}\right) \\
& =\frac{W^{\prime}(\bar{x}+\bar{y})}{W(\bar{x}+\bar{y})}-\frac{2-g}{4(\bar{x}+\bar{y})}=0 \\
\beta_{y}=\frac{\partial}{\partial y}\left(\ln \phi_{m}\right. & \left.+\ln \phi_{f}\right) \\
& =\frac{W^{\prime}(\bar{x}+\bar{y})}{W(\bar{x}+\bar{y})}-\frac{1}{2(\bar{x}+\bar{y})}=0
\end{aligned}
$$

Both (A5a) and (A5b) specify a line of slope minus 1 $(\bar{x}+\bar{y}=$ constant $)$. If $g>0$, these two lines are parallel, implying that there is no equilibrium. If $g=0$, two lines coincide, forming a line of equilibria, and the system is neutrally stable.

The total amount of growth factor $\bar{x}+\bar{y}$ that satisfies (A5a) is equal to the paternal optimum and the one satisfying $(\mathrm{A} 5 \mathrm{~b})$ is the maternal optimum (see also HAIG 1992).

\section{APPENDIX B}

Effect of deleterious mutations on structural genes: Consider a $(x, y)$-regulatory region that is linked with a wild-type structural gene. First we consider the case in which it is in a reproductive female. We need to distinguish cases according to (1) whether the female accepts a single male or two males, (2) whether the alternative allele in the same holder is wild type or mutant (denote here by + or - ), and (3) whether her mate ( $s$ ) is homozygous wild type or heterozygous (denote by $+/+$ or $+/-$ ). There is no possibility of homozygosity of mutants, which are lethal and eliminated before the reproductive stage.

Let $f$ be the gene frequency of the mutant at the reproductive stages (instead of the time of fertilization). Then the frequencies of wild-type homozygotes and heterozygotes are $1-2 f$ and $2 f$, respectively. On the other hand, the alternative allele in the same holder for a randomly chosen wild-type allele is also wild type with probability $1-2 f / 1-f$, and it is mutant with probability $f / 1-f$. 
The fitness of a female is

$\phi_{f}=(1-g)$ [ fitness when she accepts a single male ]

$+g$ [fitness when she accepts two males $]$.

(B1a)

The fitness when the female mates with a single male is the sum of four terms summarized as

[ fitness when she accepts a single male]

$$
\begin{gathered}
=\frac{T}{a}\left(( 1 - 2 f ) \left\{\frac{\frac{1-2 f}{1-f}}{\frac{y+\bar{y}}{2}+\bar{x}}+\frac{\bar{f}}{\frac{y}{2}+\bar{x}}\right.\right. \\
\times \frac{1}{2} W(y+\bar{x})+2 f\left\{\frac{\frac{1-2 f}{\frac{1-f}{y+\bar{y}}+\frac{\bar{x}}{2}}+\frac{f}{\frac{1}{2}+\frac{\bar{x}}{2}}}{2}\right\} \\
\left.\times \frac{1}{4}\{W(y+\bar{x})+W(y)\}\right) .
\end{gathered}
$$

The fitness when the female accepts two males should be classified into six cases, according to whether the female herself is homozygous or heterozygous, and to three cases: (1) both males are homozygous, (2) one male is homozygous and the other is heterozygous, and (3) both males are heterozygous.

[Fitness when she accepts two males]

$$
\begin{aligned}
& =\frac{T}{a}\left((1-2 f)^{2}\left\{\frac{\frac{1-2 f}{1-f}}{\frac{y+\bar{y}}{2}+\bar{x}}+\frac{f}{\frac{y}{2}+\bar{x}}\right\} \frac{1}{2} W(y+\bar{x})\right. \\
& +4 f(1-2 f)\left\{\frac{\frac{1-2 f}{1-f}}{\frac{y+\bar{y}}{2}+\frac{3 \bar{x}}{4}}+\frac{\frac{f}{1-f}}{\frac{y}{2}+\frac{3 \bar{x}}{4}}\right\} \\
& \times \frac{1}{8}\{3 W(y+\bar{x})+W(y)\} \\
& +(2 f)^{2}\left\{\begin{array}{c}
\frac{1-2 f}{\frac{1-f}{2}}+\frac{f}{1-f} \\
\frac{y}{2}+\frac{\bar{x}}{2}
\end{array}\right\} \\
& \times \frac{1}{4}\{W(y+\bar{x})+W(y)\}
\end{aligned}
$$

From (Bla) together with (B1b) and (B1c), we have female fitness function $\phi_{j}$.

The male fitness $\phi_{m}$ is

$\phi_{m}$

$=M[$ fitness obtained from mating a single female $]$.

The fitness obtained from mating with a single female is calculated in a similar way as the female fitness function $\phi_{f}$, by noting that it differs from female fitness when the female mates with two males. The result is

$$
\begin{aligned}
\phi_{m} & =\frac{M T}{a}(1-g)\left((1-2 f)\left\{\frac{\frac{1-2 f}{1-f}}{\frac{x+\bar{x}}{2}+\bar{y}}+\frac{\frac{f}{1-f}}{\frac{x}{2}+\bar{y}}\right\}\right. \\
& \times \frac{1}{2} W(x+\bar{y})+2 f\left\{\frac{\left.\frac{1-2 f}{\frac{1-f}{x+\bar{x}}+\frac{\bar{y}}{2}}+\frac{\frac{f}{1-f}}{\frac{x}{2}+\frac{\bar{y}}{2}}\right\}}{}\right.
\end{aligned}
$$$$
\begin{aligned}
& \left.\times \frac{1}{4}\{W(x+\bar{y})+W(x)\}\right) \\
& +\frac{M T}{a} 2 g\left(( 1 - 2 f ) \left\{\frac{(1-2 f) \frac{1-2 f}{1-f}}{\frac{x+3 \bar{x}}{4}+\bar{y}}\right.\right. \\
& +\frac{(1-2 f) \frac{f}{1-f}+2 f \frac{1-2 f}{1-f}}{\frac{\mathrm{x}+2 \bar{x}}{4}+\bar{y}}+\frac{2 f \frac{f}{1-f}}{\frac{x+\bar{x}}{4}+\bar{y}}
\end{aligned}
$$$$
\times \frac{1}{4} W(x+\bar{y})+2 f\left\{\frac{(1-2 f) \frac{1-2 f}{1-f}}{\frac{x+3 \bar{x}}{4}+\frac{\bar{y}}{2}}\right.
$$$$
\left.+\frac{(1-2 f) \frac{f}{1-f}+2 f \frac{1-2 f}{1-f}}{\frac{\mathrm{x}+2 \bar{x}}{4}+\frac{\bar{y}}{2}}+\frac{2 f \frac{f}{1-f}}{\frac{x+\bar{x}}{4}+\frac{\bar{y}}{2}}\right\}
$$

$$
\left.\times \frac{1}{8}\{W(x+\bar{y})+W(x)\}\right) .
$$




\section{APPENDIX C}

Genomic imprinting evolution for an inhibitor gene: Here, we consider a gene coding an inhibitor that decreases the growth rate of the embryo. For example, $\operatorname{Ig} f 2 r$ in mouse is suspected of being an inhibitor of $\lg f 2$ ( HAIG and GRAHAM 1991). The product of $\lg f 2 r$ gene decreases the size of the embryo and is expressed only from the maternal allele (BARLow et al. 1991).

We consider the expression level of an inhibitor gene, the product of which digests or inactivates embryonic growth factors. If an embryo receives the $\left(x_{p}, y_{p}\right)$ allele from its father and the $\left(x_{m}, y_{m}\right)$-allele from its mother, it produces an quantity of inhibitor $x_{p}+y_{m}$ in total. In contrast to the growth factor, the total quantity of the inhibitor decreases the demand for maternal resource supply and the survivorship of the embryo. The survivorship function of an embryo, denoted by $W_{i}\left(x_{p}+y_{m}\right)$, is a decreasing function of $x_{p}+y_{m}$. Suppose that the resource allocated by the mother to each offspring is

$$
a\left(F_{m}-\left(x_{p}+y_{m}\right)\right),
$$

where $F_{m}$ is the total amount of growth factor when no inhibitor is present. Then the fitness functions of the resource division model for this gene are

$\phi_{f}(x, y ; \bar{x}, \bar{y})$

$$
=\frac{T}{a\left(F_{m}-\left(\bar{x}+\frac{y+\bar{y}}{2}\right)\right)} \frac{1}{2} W_{i}(\bar{x}+y),
$$

$\phi_{m}(x, y ; \bar{x}, \bar{y})$

$$
\begin{array}{r}
=M \frac{T}{a}\left[\frac{(1-g)^{1 / 2}}{F_{m}-\left(\bar{y}+\frac{x+\bar{x}}{2}\right)}+\frac{2 g^{1 / 4}}{F_{m}-\left(\bar{y}+\frac{x+3 \bar{x}}{4}\right)}\right] \\
\times W_{i}(x+\bar{y}) . \quad(\mathrm{C} 2 \mathrm{~b})
\end{array}
$$

Substituting (1) by (C2a) and (C2b) gives us the evolutionary dynamics of the average expression levels from the paternal allele and the maternal allele. Suppose that survivorship function is illustrated in Figure $6 \mathrm{~A}$. As illustrated by the evolutionary trajectories of $(\bar{x}, \bar{y})$ in Figure 6B, the expression level from the

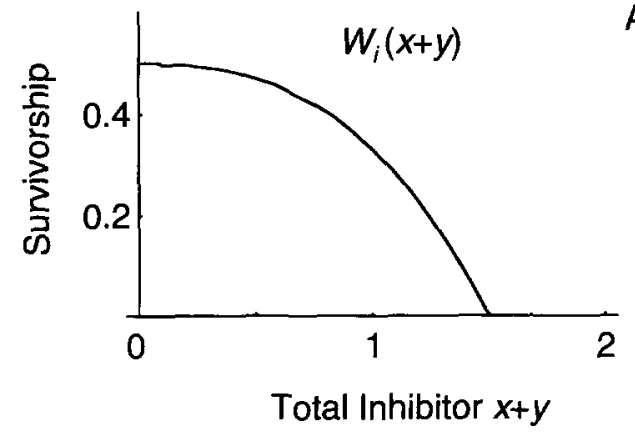

A

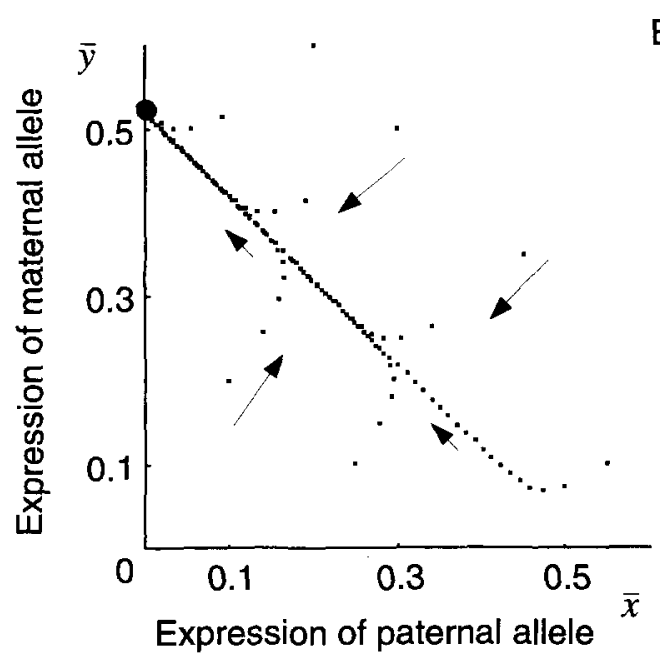

FIGURE 6.-(A) Survivorship of an embryo as a function of the amount of growth-inhibitor $z=x+y, W_{i}(z)=w_{0}\left(F_{n}\right.$ $-z-\alpha) /\left(1+\beta\left(F_{m}-z\right)^{2}\right)$, for $z<F_{m}-\alpha$, but is zero for $z>F_{m}-\alpha$. Parameters are $\alpha=0.5, \beta=0.5, F_{m}=2$ and $w_{0}$ $=1$. It decreases with $z$. Since an offspring with inhibitor higher than $F_{m}-\alpha$ dies, evolution only for the region $z<$ $F_{m}-\alpha$ is considered. (B) Evolutionary trajectories of the resource division model for a growth-inhibitor gene. Female polygamy rate is large $(g=0.3)$. Other parameters are $G_{x}=$ $G_{y}=0.2, B=0$, and $W_{i}(z)$ is given by $\mathrm{A}$. The dynamics are independent of $T, a$ and $M$. The locations of $(\bar{x}, \bar{y})$ for every other generations are indicated. There is a globally stable equilibrium on the $\bar{y}$ axis in which the paternal allele is silent.

maternal allele becomes very large and the paternal allele become silent as the result of evolution, if there is some probability for the female to accept multiple mates $(g>0)$. 